\title{
Phase-dependent X-ray observations of the $\beta$ Lyrae system
}

\section{No eclipse in the soft band}

\author{
R. Ignace ${ }^{1}$, L. M. Oskinova ${ }^{2}$, W. L. Waldron ${ }^{3}$, J. L. Hoffman ${ }^{3,4, \star, \star \star}$, and W.-R. Hamann ${ }^{2}$ \\ ${ }^{1}$ Department of Physics, Astronomy, \& Geology, East Tennessee State University, Johnson City, TN 37614, USA \\ e-mail: ignace@etsu.edu \\ ${ }^{2}$ Lehrstuhl Astrophysik der Universität Potsdam, Am Neuen Palais 10, 14469 Potsdam, Germany \\ 3 Eureka Scientific, Inc., 2452 Delmer Street Suite 100, Oakland, CA 94602-3017, USA \\ ${ }^{4}$ Department of Astronomy, UC Berkeley, 601 Campbell Hall, Berkeley, CA 94530, USA
}

Received 18 October 2007 / Accepted 26 November 2007

\section{ABSTRACT}

\begin{abstract}
Aims. We report on observations of the eclipsing and interacting binary $\beta$ Lyrae from the Suzaku X-ray telescope. This system involves an early B star embedded in an optically and geometrically thick disk that is siphoning atmospheric gases from a less massive late B II companion.

Methods. Motivated by an unpublished X-ray spectrum from the Einstein X-ray telescope suggesting unusually hard emission, we obtained time with Suzaku for pointings at three different phases within a single orbit.

Results. From the XIS detectors, the softer X-ray emission appears typical of an early-type star. What is surprising is the remarkably unchanging character of this emission, both in luminosity and in spectral shape, despite the highly asymmetric geometry of the system. We see no eclipse effect below $10 \mathrm{keV}$. The constancy of the soft emission is plausibly related to the wind of the embedded B star and Thomson scattering of X-rays in the system, although it might be due to extended shock structures arising near the accretion disk as a result of the unusually high mass-transfer rate. There is some evidence from the PIN instrument for hard emission in the 10-60 keV range. Follow-up observations with the RXTE satellite will confirm this preliminary detection.
\end{abstract}

Key words. binaries: close - binaries: eclipsing - stars: individual: $\beta$ Lyrae - X-rays: binaries

\section{Introduction}

The binary $\beta$ Lyr is a nearly edge-on, semi-detached interacting system that has undergone mass reversal and remains in a phase of large-scale mass transfer. The primary, mass-losing star (the "Loser") is a B6-B8 IIp star. The mass-gaining star (the "Gainer") is embedded in an optically thick accretion disk and is not directly visible. Although the embedded source has been considered as a possible compact object (Devinney 1971; Wilson 1971), it is probably a main sequence B0 star (Hubeny \& Plavec 1991). The system is very complex because of the bipolar jet-like structures (Harmanec et al. 1996; Hoffman et al. 1998), a circumbinary envelope (Batten \& Sahade 1973; Hack et al. 1975), and a substantial kilo-Gauss magnetic field (Leone et al. 2003).

The optical light curve of the system features a primary minimum that is $\approx 1$ magnitude deep and a secondary minimum $\approx 0.4$ mag deep (see Fig. 1); however, the secondary minimum is deeper than the primary minimum at shorter wavelengths, and below $\operatorname{Ly} \alpha$ the eclipses no longer appear (Kondo et al. 1994). A summary of the system properties is given in Table 1. The orbital period is 12.9 days, and the mean light curves appear stable with epoch. The UV spectrum of $\beta$ Lyr is dominated by an anomalous continuum and by emission lines with the unusually strong P Cygni profiles typical of hot star winds (Hack et al. 1975; Aydin et al. 1988; Mazzali 1987).

* NSF Astronomy and Astrophysics Postdoctoral Fellow.

$\star \star$ Currently at Department of Physics and Astronomy, University of Denver.

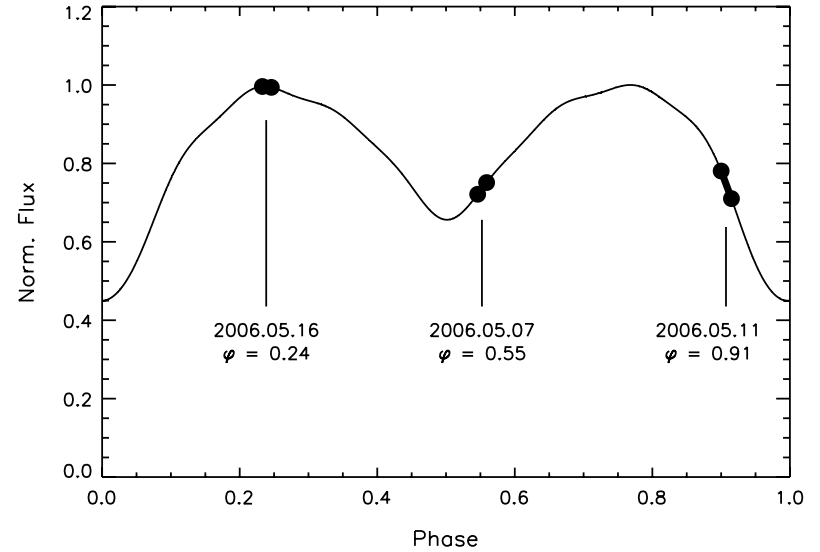

Fig. 1. Illustration of the Suzaku pointings with respect to the binary phase. The solid line represents the normalized $V$-band light curve (Fourier fit by Harmanec et al. 1996). Primary eclipse (phases 0.0 and 1.0) occurs when the mass-losing star is occulted by the disk. The three intervals marked on the curve represent our Suzaku pointings, which occurred during a single orbit in May 2006. Phases were calculated from the quadratic ephemeris of Harmanec \& Scholz (1993).

Despite numerous and ongoing modeling attempts (e.g., Wilson 1974; Linnell \& Hubeny 1996; Bisikalo et al. 2000; Linnell 2002; Nazarenko \& Glazunova 2003, 2006a,b), no model is capable yet of matching the observed light curves of $\beta$ Lyr from the IR through the UV. Strangely, $\beta$ Lyr has largely 
Table 1. Properties of $\beta$ Lyrae.

\begin{tabular}{lcc}
\hline \hline Component $^{a}$ & Gainer & Loser \\
\hline$T_{\text {eff }}[\mathrm{K}]$ & 32000 & 13300 \\
Spectral type & $\approx \mathrm{B} 0 \mathrm{~V}$ & $\mathrm{~B} 6-8 \mathrm{IIp}$ \\
$M / M_{\odot}$ & $\approx 13$ & $\approx 3$ \\
$\dot{M}^{b}\left[M_{\odot} \mathrm{yr}^{-1}\right]$ & $4.7 \times 10^{-8}$ & $7.2 \times 10^{-7}$ \\
$v_{\infty}^{b}\left[\mathrm{~km} \mathrm{~s}^{-} 1\right]$ & 1470 & 390 \\
& & \\
\hline \hline System property & & \\
\hline Orbital period & \multicolumn{2}{c}{ Value } \\
Viewing inclination & 12.9 days \\
Binary separation & \multicolumn{2}{c}{$86^{\circ}$} \\
Distance & $55-60 R_{\odot}$ \\
log $N_{\mathrm{H}}\left(\mathrm{cm}^{-2}\right)$ & $270 \mathrm{pc}$ \\
ROSAT PSPC & 20.76 \\
Einstein $\mathrm{SSS}^{d}:$ & $0.07 \mathrm{cps}$ \\
\hline
\end{tabular}

${ }^{a}$ Component and system properties taken from Harmanec (2002) unless otherwise noted.

${ }^{b}$ Mass-loss rate and terminal speed for the stellar winds of the respective binary components (Mazzali 1987).

${ }^{c}$ Berghöfer \& Schmitt (1994).

${ }^{d}$ Waldron (private comm.).

been left unstudied in the X-ray regime, despite the strong X-ray flux detected by the ROSAT HRI (Berghöfer \& Schmitt 1994). An unpublished spectrum taken with the EINSTEIN/SSS in 1979 reveals X-ray emission at relatively high energies, suggesting that phase-dependent observations may provide new clues to solving the puzzle of the $\beta$ Lyr geometry and interactions. Exploiting Suzaku's excellent sensitivity to hard X-rays, we conducted three pointed observations of $\beta$ Lyr within the same orbit. In the following section the observations and data reduction are explained in detail. Analyses of the spectra with phase are described in Sect. 3, and a discussion of the results is presented in Sect. 4.

\section{Observations and data reduction}

The joint Japan/US X-ray astronomy satellite Suzaku (Mitsuda et al. 2007) observed $\beta$ Lyr in May 2006 on three occasions at orbital phases as shown in Fig. 1, with corresponding viewing perspectives of the $\beta$ Lyr system illustrated in Fig. 2. (Note that the primary minimum occurs when the loser star is eclipsed, and the secondary minimum when the disk component is eclipsed.) The pointings were spaced approximately 4.3 days apart to sample the full 12.9 day orbit of the binary. Exposure times and count rates are tabulated in Table 2.

Suzaku carries four X-ray Imaging Spectrometers (XIS; Koyama et al. 2007) and a collimated Hard X-ray Detector (HXD; Takahashi et al. 2007). The field-of-view (FOV) for the XIS detectors is $17^{\prime} \times 17^{\prime}$. One of the XIS detectors (XIS1) is back-side illuminated (BI) and the three other (XIS0, XIS2, and XIS3) are front-side illuminated (FI). The bandpasses are $\sim 0.4-12 \mathrm{keV}$ for the FI detectors and $\sim 0.2-12 \mathrm{keV}$ for the BI detector. The BI CCD has higher effective area at low energies, but its background level across the entire bandpass is higher compared to the FI CCDs.

The angular resolution of the X-ray telescope onboard Suzaku is $\approx 2^{\prime}$; therefore, in the XIS image, $\beta$ Lyr is not resolved from two nearby B-type stars: HD $174664(\beta$ Lyr B) and HD 174639. While the latter star was not detected by ROSAT, the former has a ROSAT HRI count rate of $4 \times 10^{-3} \mathrm{cps}$, as compared to the $\beta$ Lyr ROSAT HRI count rate $4 \times 10^{-2} \mathrm{cps}$. We are

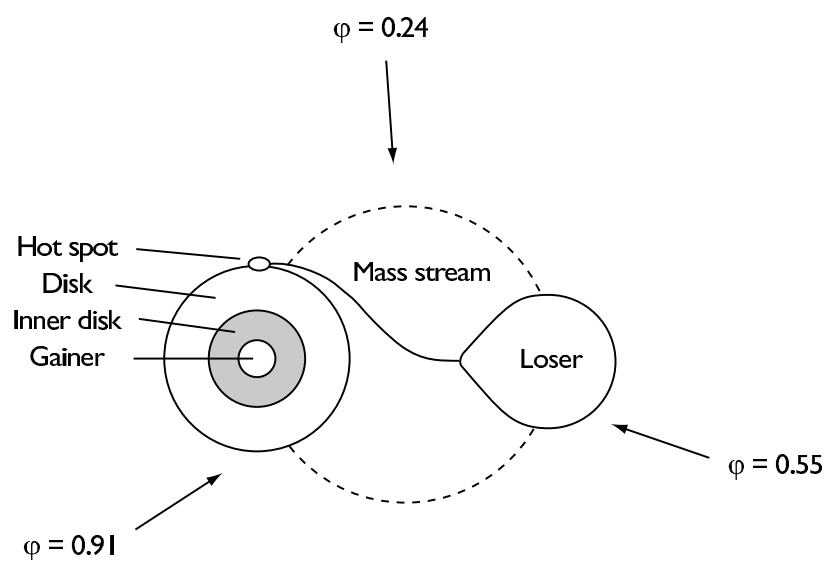

Fig. 2. Graphic topview representation of the $\beta$ Lyr star-disk system, following the model of Hoffman et al. (1998). Components are illustrative and not to scale. The three Suzaku pointings occurred at phases $\varphi=0.24,0.55$, and 0.91 all within the same orbit. Arrows indicate the Earth's line-of-sight for these three phases.

confident that the X-ray flux detected by Suzaku is at least $90 \%$ dominated by $\beta$ Lyr.

The HXD consists of two non-imaging instruments (the PIN and GSO; see Takahashi et al. 2007) with bandpasses of $\sim 10-70 \mathrm{keV}$ (PIN) and $\sim 40-600 \mathrm{keV}$ (GSO), and with an FOV of $34^{\prime} \times 34^{\prime}(\mathrm{PIN})$. Both of the HXD instruments are background-limited. The background subtraction for the HXD is performed by modeling the background spectrum. Presently, the non-X-ray background model (e.g., particle events) is known for the PIN detector with $\sim 3-5 \%$ accuracy (Kokubun et al. 2007). The background modeling for GSO data is currently far less certain than for the PIN, so we do not report on the GSO measurements.

HXD-PIN data reduction and extraction of spectra were performed using the latest calibration sources and background models. We account for cosmic X-ray background (CXB) in fitting the spectral models using the procedure suggested by the Suzaku team based on a "typical" CXB spectrum (see www . astro.isas . ac. jp/suzaku). We do not correct for contributions to the HXD background due to the South Atlantic Anomaly (SAA; see Kokubun et al. 2007) because our observations of $\beta$ Lyr were performed when the background count rate was at its lowest.

Based on the Rosat All-Sky Survey (RASS), there are a few X-ray sources in the $34^{\prime} \times 34^{\prime}$ PIN's field of view (FOV); however, our target $\beta$ Lyr is by far the brightest. The stellar coronal X-ray sources present in the FOV are expected to be faint in the HXD's energy range. There is also an active galactic nucleus (AGN) in the FOV about $15^{\prime}$ away from $\beta$ Lyr. This AGN, QSO B1847+3330, is cataloged in the RASS with a count rate of $0.05 \mathrm{cps}$. To estimate its potential contribution to the 10 $70 \mathrm{keV}$ energy range, we adopted a standard AGN power-law spectrum with $\Gamma=2$ and a low interstellar absorption column of $N_{\mathrm{H}}=5 \times 10^{20} \mathrm{~cm}^{-2}$. The predicted count rate for the Suzaku $\mathrm{PIN}$ is $\approx 0.006 \mathrm{cps}$. The observed HXD-PIN count rates are listed in Table 3. The contribution of QSOB1847+3330 is between $10-30 \%$ of the detected flux.

\section{Analysis}

A comparison of our three XIS spectra below $10 \mathrm{keV}$ with the XIS detectors indicates very little variability with phase (see Fig. 3). Based on chi-square model fits to the data, there is no 
Table 2. XIS observations of $\beta$ Lyrae.

\begin{tabular}{|c|c|c|c|c|c|c|}
\hline \multirow{2}{*}{ Date } & \multirow{2}{*}{$\varphi^{a}$} & \multirow{2}{*}{$\begin{array}{c}\text { Exposure } \\
(\mathrm{ks})\end{array}$} & \multicolumn{4}{|c|}{ XIS count rate $\left(10^{2} \mathrm{cps}\right)$} \\
\hline & & & XIS0 & XIS1 & XIS2 & XIS3 \\
\hline 2006 May 7 & 0.55 & 15.4 & $6.35 \pm 0.24$ & $8.53 \pm 0.36$ & $6.33 \pm 0.24$ & $5.15 \pm 0.22$ \\
\hline 2006 May 12 & 0.91 & 17.7 & $5.76 \pm 0.21$ & $8.14 \pm 0.33$ & $5.56 \pm 0.21$ & $4.86 \pm 0.20$ \\
\hline 2006 May 16 & 0.24 & 15.7 & $6.15 \pm 0.23$ & $9.58 \pm 0.36$ & $6.23 \pm 0.22$ & $4.93 \pm 0.22$ \\
\hline
\end{tabular}

${ }^{a}$ The orbital phases for $\beta$ Lyr were computed at the midpoint of each observation using the quadratic ephemeris of Harmanec \& Scholz (1993). Phases 0.0 and 1.0 correspond to primary eclipse.

Table 3. PIN observations of $\beta$ Lyrae.

\begin{tabular}{cccc}
\hline \hline$\varphi^{a}$ & $\begin{array}{c}\text { Exposure } \\
(\mathrm{ks})\end{array}$ & $\begin{array}{c}\text { Count rate } \\
(\mathrm{cps})\end{array}$ & $\begin{array}{c}\text { Background } \\
(\mathrm{cps})\end{array}$ \\
\hline 0.55 & 14.7 & $0.623 \pm 0.007$ & $0.536 \pm 0.002$ \\
0.91 & 17.0 & $0.505 \pm 0.005$ & $0.509 \pm 0.002$ \\
0.24 & 14.1 & $0.573 \pm 0.006$ & $0.564 \pm 0.002$ \\
\hline
\end{tabular}

evidence of statistically significant differences between the three spectra. For illustration a model fit for the $\varphi=0.24$ pointing appears as the solid line in each of the three panels of Fig. 3; its good agreement with the data at all phases demonstrates that the soft X-ray spectrum of $\beta$ Lyr is nearly constant in shape and strength. Independent fits for each pointing are very similar.

These XIS X-ray spectra are most probably thermal in nature, since emission lines are detected at $1.35 \mathrm{keV}$ (Mg XI) and $1.86 \mathrm{keV}$ (Si XIII). Our fits indicate a two-component model with the majority of the XIS X-ray emission arising from a temperature of $\approx 7.2 \mathrm{MK}$ and a hotter but much weaker component of $\gtrsim 20 \mathrm{MK}$. Solar abundances were adopted, except for nitrogen that was enhanced by more than 10 times to achieve an adequate fit. The derived hydrogen column density from the model is $N_{\mathrm{H}}=6.5 \pm 0.24 \times 10^{20} \mathrm{~cm}^{-2}$, largely consistent with the interstellar value.

Hard emission in the $10-60 \mathrm{keV}$ band of the PIN is background-dominated. The observed total and the background count rates are given in Table 3 . The source counts come from the difference of the total and background values. Based on the total counts, there is a significant source detection at phase $\varphi=0.55$, a non-detection for $\varphi=0.91$, and a marginal detection at $\varphi=0.24$. Emission at such hard energies is exceptionally unusual and unexpected for a system like $\beta$ Lyr that consists of two early-type stars. The implied hard X-ray luminosity above $10 \mathrm{keV}$ at $\varphi \approx 0.55$ is about $10^{-3} L_{\odot}$. If confirmed, this hard component would be an important tracer of the flow geometry and shock structure since the circumstellar material is quite transparent to such high-energy X-rays.

\section{Discussion}

The results of our Suzaku study are both revealing and perplexing. We had expected to see an eclipse of soft X-rays and essentially no hard emission. Instead, we found that the soft X-rays were nearly constant, and there is an indication of a very hard emission component in the system. The near constancy of the soft spectrum suggests that the X-ray source must be axially symmetric. This is quite surprising, since the system is intrinsically non-axisymmetric.

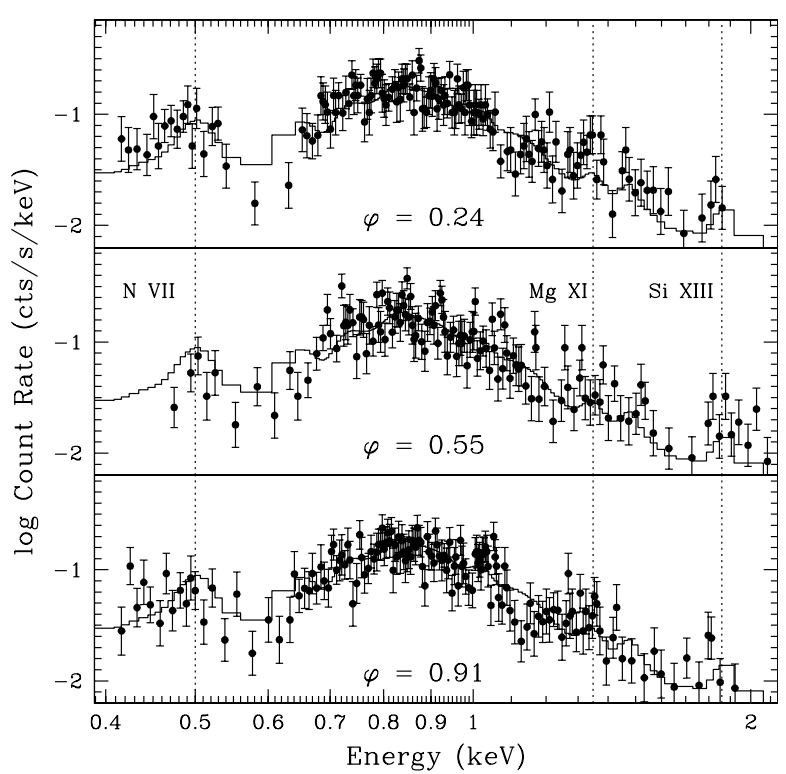

Fig. 3. Spectra of $\beta$ Lyr from the XIS1 detector for each pointing. Phase within the orbit is indicated in each panel. The data have reasonably high signal-to-noise, and it is clear that the spectral distribution shows little change with phase, because of the nearly constant brightness and spectral shape. The solid line is a model fit to the data of $\varphi=0.24$ that is replotted in the other phases for comparison.

It is worth recalling that the UV lightcurve below Ly $\alpha$ is notable for lacking any eclipses as well (Kondo et al. 1994). A somewhat analogous source to $\beta$ Lyr is the near edge-on interacting binary W Ser. Weiland et al. (1995) interpret the UV spectra of W Ser in terms of an extended boundary layer between the star and the accretion disk. Thus, it may be possible that the largely steady FUV and soft X-ray emission could arise from a similar region in the $\beta$ Lyr system. However, we do not currently favor this explanation since the most recent modeling of the disk and star components of $\beta$ Lyr (Linnell 2002) indicate that gainer star is entirely obscured at all phases.

An alternative view is suggested by hydrodynamic simulations. Bisikalo et al. (2000) modeled the mass transfer of $\beta$ Lyr and found that they could reproduce a disk-like structure but without a well-identified "hot spot". Instead, their simulations yielded a complex distribution of extended shocks. They also were able to generate a bipolar flow similar to the jet detected by Harmanec et al. (1996) and Hoffman et al. (1998). However, radiative cooling was not treated self-consistently in those simulations, but approximated through the equation of state. It is unclear whether this model can generate gas at temperatures high enough to produce the observed X-rays. Hydrodynamic modeling of the $\beta$ Lyr system has also been pursued by 
Nazarenko \& Glazunova (2003, 2006a,b), first in 2D simulations and then in $3 \mathrm{D}$ simulations. These researchers included cooling curves in their simulations and allowed for a stellar wind by the gainer. Their models also lead to a disk, a bipolar flow, and an environment permeated with elongated shocks at different azimuthal orientations about the gainer. "Warm" gas temperatures up to about $200000 \mathrm{~K}$ are achieved, but such gas cannot contribute significant emission to the X-ray band. The gas dynamical simulations are qualitatively promising in terms of predicting shocked structures that are spatially distributed in radius and azimuth throughout the disk. In such a model, the hot gas could be viewable potentially at every phase. However, X-ray emissions were not the focus of those studies, and it would be useful to have new simulations that emphasize the hot plasma structures.

We prefer an interpretation in which the X-rays arise from distributed shocks in the wind of the gainer star. At $k T \approx$ $0.6 \mathrm{keV}$, the spectral characteristics of the Suzaku spectra are compatible with a typical early main sequence B star, and the XIS luminosity $L_{X}(\mathrm{XIS}) \approx 6.6 \times 10^{30} \mathrm{erg} \mathrm{s}^{-1}$ is commensurate with the soft emission expected from an early B0-B1 star (Cohen et al. 1997). Although the favored model for the binary geometry may preclude a direct view of the gainer star, it does permit a reasonably deep view into its wind, even at secondary eclipse (with the giant star at front). Moreover, we know from the polarimetric study by Hoffman et al. (1998) that there is substantial scattering opacity above and below the disk plane. The near constancy of the X-rays seen with the XIS may result from an extended "halo" of scattered X-ray light, similar in spirit to the scattered optical light observed in images of some Herbig-Haro objects seen edge-on to their disks, such as HH 30 (Burrows et al. 1996). In this picture the soft X-rays ultimately originate in the shocked wind of the early gainer star. Part of the emission is observed directly from hot plasma at large radii in the wind, and part is scattered into our line-of-sight from above and below the disk. Thus the $0.6 \mathrm{keV}$ temperature of the hot gas would represent an upper limit value owing to the fact that photoabsorption of X-rays is more severe toward the soft end of the spectrum. On the other hand, the observed X-ray luminosity must be a lower limit.

We have considered three distinct origins for the observed $\mathrm{X}$-ray emissions. At this point we favor the gainer wind as the source of the soft X-rays, but acknowledge that future hydrodynamic modeling may change this picture. In addition, there seems to be an unusually hard component of emission in the system, in excess of $10 \mathrm{keV}$. Such hard emission is more typically associated with compact objects, and not with the winds of hot stars (e.g., Berghöfer et al. 1997). However, the nature of the gainer is still ambiguous; perhaps the X-ray properties of $\beta$ Lyr could be understood in relation to a central compact object (Devinney 1971; Wilson 1971). Although this seems unlikely, the X-ray observations certainly do not conform to our original expectations, and so at least a reconsideration of the possibility seems in order. The X-ray pointings by Suzaku offer the tantalizing promise of providing new and valuable information about the $\beta$ Lyr system, but it is clear that a far more rigorous sampling of the X-ray lightcurve and source spectrum and new models for the system will be needed to test the suppositions that we have put forth. We have obtained time with the RXTE satellite to create a more complete X-ray light curve of $\beta$ Lyr, which should provide new insight into this complex system in the near future.

Acknowledgements. We wish to thank the referee Ed Devinney, an anonymous referee, and Steve Shore for several valuable comments. We are grateful for advice given by Hugh Hudson in relation to the background of hard X-rays and by Georg Lamer in relation to the X-ray spectra of QSOs. We also thank Koji Mukai and Nick White for technical assistance regarding Suzaku. R.I. received support from NASA grant award NNX06AI04G. LMO acknowledges support by the Deutsche Forschungsgemeinschaft with grant Fe 573/3. W.L.W. was supported in part by NASA contract NNG07EF47P. JLH acknowledges the support of a NSF Astronomy \& Astrophysics Postdoctoral Fellowship under award AST0302123 .

\section{References}

Aydin, C., Brandi, E., Engin, S., et al. 1988, A\&A, 193, 202

Batten, A. H., \& Sahade, J. 1973, PASP, 85, 599

Berghöfer, T. W., \& Schmitt, J. H. M. M. 1994, A\&A, 292, L5

Berghöfer, T. W., Schmitt, J. H. M. M., Danner, R., \& Cassinelli, J. P. 1997, A\&A, 322, 167

Bisikalo, D. V., Harmanec, P., Boyarchuk, A. A., Kuznetsov, O. A., \& Hadrava, P. 2000, A\&A, 353, 1009

Burrows, C. J., Stapelfeld, K. R., Watson, A. M., et al. 1996, ApJ, 473, 437

Cohen, D. H., Cassinelli, J. P., \& MacFarlane, J. J. 1997, ApJ, 487, 867

Devinney, E. J. 1971, Nature, 233, 110

Hack, M., Hutchings, J. B., Kondo, Y., et al. 1975, ApJS, 34, 565

Harmanec, P. 2002, Astron. Nachr., 323(2), 87

Harmanec, P., Morand, F., Bonneau, D., et al.1996, A\&A, 312, 879

Harmanec, P., \& Scholz, G. 1993, A\&A, 279,131

Hoffman, J. L., Nordsieck, K. H., \& Fox, G. K., 1998, AJ, 115, 1576

Hubeny, I., \& Plavec, M. J. 1991, AJ, 102, 1156

Kokubun, M. et al. 2007, Publ. Astron. Soc. Japan 59, S53

Kondo, Y., McCluskey, G. E., Sivis, J. M. S., et al. 1994, ApJ, 421, 787

Koyama, K., et al. 2007, Publ. Astron. Soc. Japan 59, S27

Leone, F., Plachinda, S. I., Umana, G., Trigilio, C., \& Skulsky, M. 2003, A\&A, 405,223

Linnell, A. P. 2002, MNRAS, 334, 963

Linnell, A. P., \& Hubeny, I. 1996, ApJ, 471, 958

Mazzali, P. A. 1987, ApJS, 65, 695

Mitsuda, K., Bautz, M., Inoue, H. et al.2007, Publ. Astron. Soc. Japan 59, S1

Nazarenko, V. V., \& Glazunova, L. V. 2003, Astr. Rep., 7, 1013

Nazarenko, V. V., \& Glazunova, L. V. 2006a, Astr. Rep., 50, 369

Nazarenko, V. V., \& Glazunova, L. V. 2006b, Astr. Rep., 50, 380

Takahashi, T., Abe, K., Manabu, E., et al. 2007, Publ. Astron. Soc. Japan 59, S35

Weiland, J. L., Shore, S. N., Beaver, E. A., Lyons, R. W., \& Rosenblatt, E. I. 1995, ApJ, 447, 401

Wilson, R. E. 1971, Nature, 234, 406

Wilson, R. E. 1974, ApJ, 189, 319 